\title{
Calidad de vida en mujeres con alteraciones del piso pélvico: revisión de la literatura
}

\author{
Claudia Flores E. 1 , Javier Pizarro $B^{2}$ \\ 1 Enfermera Matrona, Departamento de Salud de la Mujer, Escuela de Enfermería, Pontificia Universidad Católica de \\ Chile. ${ }^{2}$ Servicio de Obstetricia y Ginecología, Dr. Sótero del Río, División de Obstetricia y Ginecología, Escuela de Me- \\ dicina, Pontificia Universidad Católica de Chile.
}

a Enfermera Matrona.

\section{RESUMEN}

Antecedentes: El riesgo de desarrollar prolapso genital aumenta con la edad, con un efecto negativo en la calidad de vida. Objetivo: Describir el efecto de los trastornos del piso pélvico en la calidad de vida en las distintas dimensiones, sin mediar intervenciones. Método: Se realizó una búsqueda en las bases de datos Pubmed, Cinhal, Proquest y Scielo durante mayo de 2011. Resultados: Los síntomas generan un gran impacto en la calidad de vida afectando la función sexual, actividades cotidianas y generando efectos negativos en aspectos psicosociales, como la imagen corporal y el desempeño de las mujeres. Conclusión: Es necesario generar una atención integral centrada en síntomas, función sexual, educación y apoyo emocional.

\section{PALABRAS CLAVE: Prolapso del tracto vaginal, incontinencia urinaria, calidad de vida}

\section{SUMMARY}

Background: The risk of develop genital prolapse increases with age, with a negative effect in quality of life. Objective: The aim of this review is to describe the effect of pelvic floor disorders on quality of life in the different dimensions in women, without any interventions. Method: It developed a review in Pubmed, Cinhal, Proquest and Scielo database during may of 2011. Results: The symptoms produce a great impact on quality of life affecting sexual function, daily activities and it produce negative psychosocial effects like body image and women's performance. Conclusion: Must be necessary generate a integral attention to women, focused on symptoms, sexual function, education and emotional support.

\section{KEY WORD: Genital tract prolapse, urine incontinence, quality of life}

\section{INTRODUCCIÓN}

El prolapso genital ha sido definido como el descenso de una o más de las paredes vaginales, cuello del útero, o cúpula vaginal. La presencia de estos signos, podría estar relacionada con síntomas de prolapso. Por otro lado, se ha definido in- continencia de orina (IO) como la observación de pérdida involuntaria de orina durante el examen físico (1).

El riesgo de desarrollar prolapso aumenta con la edad (2). Se estima que entre un 3 a $6 \%$ de la población femenina desarrollará prolapso severo en algún momento de su vida (3). Así mismo, el 
riesgo de necesitar cirugía por estas patologías es de un $11 \%$ (4).

La calidad de vida (CV) de las mujeres se ve afectada, independiente del grado de severidad del prolapso $(3,5,6)$. La International Continence Society (ICS) y la International Urogynecology Association (IUGA), recomiendan la inclusión de medición de calidad de vida en todos los estudios de incontinencia urinaria o prolapso, evidenciando su evaluación como un resultado esencial después de cualquier intervención en el área (7).

En los últimos años el éxito de las cirugías de piso pelviano ha sido evaluado utilizando instrumentos de medición de calidad de vida específicos y otros generales no específicos (8-12). El entendimiento del efecto global de las alteraciones de piso pélvico en las mujeres afectadas, es fundamental para entender estas condiciones de manera integral. Sin embargo, no existe consenso de cual es el instrumento ideal a utilizar para evaluar CV en pacientes con prolapso genital. Esto ha generado múltiples formas de reportar CV disminuyendo la capacidad comparativa o validez externa de los trabajos.

El objetivo de esta revisión es describir el efecto de los trastornos del piso pélvico en la CV de las mujeres en sus distintas dimensiones, sin mediar intervenciones. Se espera con esto, contribuir al conocimiento en el tema de modo de incentivar un abordaje de esta problemática de manera óptima.

\section{MÉTODO}

Se realizó una búsqueda en las bases de datos Pubmed, Cinhal, Proquest y Scielo durante el mes de mayo de 2011. La estrategia de búsqueda incluyó las palabras claves: quality of life and pelvic organ prolapse, quality of life and prolapse, quality of life and urinary incontinence y health related quality of life and pelvic organ prolapse, y sus respectivas traducciones al español para el buscador Scielo. Para realizar la búsqueda se especificaron los siguientes límites: artículos en idioma inglés, español o portugués, publicados desde el año 2005 en adelante.

Se encontraron 949 artículos, de los cuales, se seleccionaron únicamente aquellas investigaciones que no consideraran intervenciones médicas o quirúrgicas, dando un total de 31 artículos.

Los resultados de la revisión incluyen: descripción de los instrumentos utilizados, diferencias en la CV de acuerdo a los síntomas presentados, efectos en la CV según etapa del ciclo vital y efectos en los ámbitos psicosociales.

\section{RESULTADOS}

Los artículos encontrados entregan evidencia de tipo cuantitativa y cualitativa, lo que permite una mejor comprensión de la CV. En este sentido, 30/31 trabajos analizados, utilizaron instrumentos específicos, ya validados, solo un artículo muestra un cuestionario creado para dicho propósito (13).

Las características de los instrumentos es diversa, por lo que surge la necesidad de describir cada uno de ellos antes de analizar la información:

\section{A. Instrumentos utilizados para evaluar calidad de vida}

Se realizó una división de acuerdo a la dimensión particular que evalúan (Tabla I): 1. Percepción de salud; 2. Evaluación de incontinencia urinaria; 3. Síntomas de piso pelviano; y 4 . Sexualidad.

Tabla I

DESCRIPCIÓN DE INSTRUMENTOS UTILIZADOS

\begin{tabular}{ll}
\hline TEMA & NOMBRE \\
\hline $\begin{array}{l}\text { Percepción de } \\
\text { salud }\end{array}$ & $\begin{array}{l}\text { Health Survey (SF_36) o Short } \\
\text { Health Form Survey (SF_12) }\end{array}$
\end{tabular}

MEDICIÓN

salud

Instrumento genérico que evalúa calidad de vida asociada a la salud y aspectos físicos y emocionales. Contiene 8 dimensiones: función física; limitación del rol por aspectos físicos y emocionales; dolor físico; percepción general de salud; energía y vitalidad; función social y salud mental $(32,38,47)$.

Nottingham Health Profile

Instrumento genérico para evaluar diferentes condiciones médicas. Contiene 38 preguntas que se dividen en 6 dimensiones: movilidad física, dolor, reacciones emocionales, aislamiento social, energía y sueño (30). 
Patient Health Questionnaire (PHQ-9)

Incontinencia de orina

King's Health Questionnaire (KHQ)

Incontinence questionnaire Short Form (ICQ-SF)

International Consultation on Incontinence-Urinary Incontinence Short Form (ICIQ-UI SF)

Wagner`s Quality of Life Scale

Incontinence Impact Questionnaire (IIQ)

Sandvik Severity Scale

Incontinence QOL (I-QOL)

Incontinence Severity Index

Pelvic Floor Distress Inventory (PFDI)

Síntomas de piso pélvico

Prolapse Quality of life

(P_QOL)

Pelvic Floor impact

Questionnaire (PFIQ)
Mide síntomas depresivos, indicando su severidad y correlaciona con diagnóstico de depresión mayor (44).

Evalúa 9 dimensiones: Percepción de salud general y 8 dominios de calidad de vida: Impacto de la incontinencia, Limitación del rol, Limitación física, Limitación social, Relaciones personales, Emociones, Alteraciones de sueño/ energía, Severidad del cuadro $(19-21,24,33,40,48)$.

Tiene 4 secciones, las primeras dos evalúan frecuencia y severidad de las pérdidas de orina, la tercera parte evalúa calidad de vida y cuarta evalúa tipo de incontinencia (36).

Tiene 2 grupos de preguntas, la primera en relación a alteración de la calidad de vida y la segunda orientadas a definir el tipo de 10 (40).

Consiste en 28 preguntas para mujeres embarazadas sobre incontinencia, actividades diarias y características psicosociales (36).

30 preguntas de selección múltiple que evalúa 4 dominios: actividad física, salud emocional, relaciones sociales y viaje $(15,23,31,32,37)$.

Evalúa la severidad clínica de la incontinencia, mide frecuencia y cantidad de orina perdida(15).

Contiene 22 ítems divididos en 3 subescalas: evitación y limitación de la conducta, impacto psicosocial y vergüenza social (22).

Evalúa severidad de la $I O$ según frecuencia y cantidad de las pérdidas de orina (22).

Evalúa únicamente síntomas. Se ordenan en 3 subescalas: Alteración urinaria (UDI), alteración por el prolapsoPOPD), alteraciones colo-rectales (CRADI). Algunos estudios incorporaron solo algunas de estas subescalas $(14,16,18,23,25,26,29-31,33,36,43)$.

Es similar al UDI, puede ser usado para evaluar la presencia y molestias de los síntomas de defecación (28).

Evalúa 9 dominios: salud general, impacto del prolapso, limitación del rol, física, social, relaciones personales, emociones, sueño/energía y severidad de los síntomas $(17,27)$.

Evalúa actividades del diario vivir y como estas afectan los síntomas vesicales, intestinales o pélvicos, midiendo las sensaciones o sentimientos que provocan, se divide en 3 escalas: Impacto de la incontinencia, impacto colorectal e impacto del prolapso pélvico $(14,15,28,44)$.

Sexualidad

Pelvic Organ

Prolapse/Urinary

Incontinence Sexual Function

Corresponde a la versión corta del mismo nombre. Mide función sexual en 3 factores: Comportamiento/emociones, aspectos físicos y relación de pareja (15). 
Female Sexual Function

Index FSFI)

Vaginal Changes Sexual and Body Image Steem Scale

Bristol Female Lower Urinary Tract Symptoms questionnaire (BFLUTS)

Body Exposure During Sexual Activities Questionnaire (BESAQ)
No es específico para piso pélvico, contiene seis domnios: deseo, excitación, lubricación, orgasmo, satisfacción y dolor (37).

Consiste en 10 preguntas que evalúan grado de acuerdo con aseveraciones respecto de la sexualidad relacionada a los cambios producidos por el prolapso (40).

Evalúa síntomas y el impacto en la función sexual y calidad de vida asociada a la salud. Son 8 ítems relacionados a IO, 12 evalúan otros síntomas, 4 asociados al almacenamiento y eliminación de orina. 9 ítems evalúan aspectos específicos de la CV y 4 ítems de función sexual (38).

28 preguntas para evaluar ansiedad, rechazo corporal, principalmente asociado a actividad sexual o contacto(36).

\section{B. Efectos en la calidad de vida}

1. Diferencias de acuerdo a los síntomas. Los síntomas de prolapso e incontinencia son el aspecto mayormente estudiado. La mayoría de los artículos revisados describen las diferencias en la severidad de síntomas según los tipos de incontinencia o el grado de prolapso. Tres artículos revisados concuerdan que el efecto global en la CV es moderado sin importar el grado de prolapso (14-16). Los estudios cualitativos, concluyen que las mujeres podrían estar más afectadas que lo que logran objetivar estos instrumentos $(15,17)$.

Los síntomas de incontinencia de orina mencionados son: frecuencia miccional aumentada, urgencia miccional, nicturia e incontinencia urinaria de esfuerzo (18-20). El impacto en la CV se describe como moderado a alto; este efecto se visualiza en diversas dimensiones destacando las limitaciones personales como actividad física y limitaciones del rol como realizar tareas domésticas (21). A mayor cantidad o severidad de los síntomas de incontinencia la CV es peor $(22,23)$. Al separar por tipo de IO, observamos que la mixta que incluye incontinencia de esfuerzo así como la incontinencia de urgencia (IOU) y urgencia miccional, demuestra tener peores efectos en la CV de las mujeres $(18,19,24,25)$. Así mismo, según el síntoma de prevalencia en la incontinencia mixta, los síntomas de IOU generan mayor impacto en la CV en comparación con los síntomas de esfuerzo (24).

En cuanto a incontinencia fecal, la literatura muestra que estas mujeres además tienen mayores síntomas urinarios por el prolapso, no obstante el impacto en la vida es similar a las que no tienen incontinencia fecal (26). Las personas afectadas por prolapso, reportan dificultad para defecar, dolor abdominal y dificultad para orinar. La frecuencia de los síntomas se asocia con peor CV, afectando la movilidad, dolor y energía de manera estadísticamente significativa. Se describen otros efectos como reacciones emocionales ansiedad y estrés, aislamiento social y alteración del sueño sin alcanzar significancia estadística $(14,15,27)$.

Así mismo, al comparar los síntomas con los cambios en la fuerza muscular del piso pélvico, no existen diferencias significativas entre grupos sintomáticos y asintomáticos $(14,28)$, se describen mayores síntomas urinarios y molestias en aquellas con menor fuerza muscular (14).

En relación a diferencias étnicas, para prolapso o IO, las mujeres de raza negra tienen mayores síntomas de IOU y las de raza blanca y latinas mayores síntomas de incontinencia de esfuerzo (IOE) (22). Un estudio demostró que las mujeres negras tenían mayor fuerza muscular que las blancas lo que podría explicar la diferencia en los síntomas (29).

La obesidad se ha descrito como un factor de riesgo para desarrollar alteraciones del piso pélvico, cinco estudios concuerdan en que a mayor índice de masa corporal, existen mayores síntomas y alteración en la calidad de vida $(17,23,25,30,31)$. La obesidad empeora la calidad de vida de las mujeres de raza blanca, pero no así para las de raza negra (23). 2. Efectos según etapa del ciclo vital. En relación a prolapso genital, el grado de severidad tiene directa relación con la edad de las mujeres, siendo más severo a mayor edad (16). Las alteraciones del prolapso se producen principalmente en la adultez media y mayor. 
Un estudio reportó que las mujeres con menor edad en la muestra reportaron peor calidad de vida que las mayores (23). Así mismo, los grupos con menor edad, reportaron estar más preocupadas por los aspectos físicos, en cambio las mayores por los aspectos sociales (15).

En la adultez media, la CV es peor mientras más síntomas se tengan, destacando dificultad en la movilidad, dolor, reacciones emocionales negativas, y alteración en la energía (27). Así mismo, se ha descrito mayor afectación en el nivel de bienestar en mujeres con IOU comparadas con mujeres con IOE para este grupo etáreo (32).

En estudios realizados en residencias de larga estadía para adultos mayores, es interesante destacar que para ellos, el tener incontinencia forma parte de su vida, sin considerarlo un factor que altere la CV $(33,34)$, plantean además, que las mujeres refieren un "acostumbramiento" frente a estas condiciones, sin considerarlo un aspecto negativo (34). Los adultos mayores generan cambios en sus actividades diarias para tener una vida más cómoda (33).

Respecto del embarazo, se ha descrito que predominan los síntomas de IOE, sobretodo en aquellas mujeres que tuvieron incontinencia en embarazos previos. Los síntomas son más frecuentes en el tercer trimestre y el impacto en la CV es considerado como mínimo $(35,36)$. A pesar de que la CV no se ve afectada mayormente, la imagen corporal de la embarazada es peor a medida que se aumenta de peso, no obstante, el peso por sí solo no es indicativo de peor imagen corporal incluyendo la etapa de postparto. Durante la etapa posparto se encuentra afectada la dimensión de depresión y autopercepción, además de sentirse menos atractivas para sus parejas (36).

3. Efectos en los ámbitos psicosociales. En este tema se analizarán los resultados respecto de la sexualidad, actividades cotidianas y aspectos psicológicos.

Sexualidad. En algunos grupos el impacto se ha descrito como moderado; se describen molestias en relación a la sensación de obstrucción durante la penetración, así como laxitud vaginal que genera disminución de la satisfacción sexual. Las mujeres con prolapso en etapas avanzadas (III o IV) tienen mayor probabilidad de sentirse cohibidas, menos femeninas y atractivas sexualmente que aquellas mujeres sin prolapso, además de tener dificultad en mantener algunas posiciones durante la actividad sexual (36-38), así mismo, mujeres con incontinencia describen sentirse menos deseables y describen preocupación por la respuesta de sus parejas frente al prolapso $(37,38)$.

Aquellas mujeres con incontinencia durante la actividad sexual, describen mayor impacto en la CV en la dimensión de relaciones personales, lo que se relaciona con su pareja y vida sexual en comparación con mujeres con otros síntomas urinarios pero sin incontinencia coital $(13,19,21)$, ellas describen su afectación como moderada o grave (36). Las mujeres con pérdidas de orina durante el coito tienen con mayor frecuencia síntomas de IOE (19), no obstante aquellas con IOU pierden orina más frecuentemente que las mujeres con vejiga hiperactiva y manifiestan mayor dolor (39).

Respecto del origen de la incontinencia, según algunas mujeres se encuentra en historias negativas del pasado, como violaciones o relaciones sexuales con su pareja sin consentimiento (40).

Actividades cotidianas. El prolapso y la incontinencia han demostrado generar un impacto negativo en el trabajo $(13,15)$, así como en las actividades cotidianas en el hogar $(15,17)$. Respecto de las actividades sociales y físicas, las mujeres describen dificultad para hacer actividad física y esto genera preocupación por aumentar de peso y sentirse incompetentes, así como preocupaciones por salir de vacaciones o reunirse con amigos $(13,15)$. Además, las mujeres con prolapso describen una peor imagen corporal que se relaciona de manera moderada con peor $\mathrm{CV}$ en aspectos físicos y mentales (41).

En relación a las alteraciones en las actividades diarias, las adultas mayores describen restricción en la ingesta de líquidos, así como buscar proximidad y disponibilidad de baños, lo que perjudica las actividades fuera de la casa $(34,42)$. En cuanto a la ropa que usan, describen calzones firmes, toallas o pañales y ropa oscura $(33,34)$, al ser la incontinencia vista como algo natural, estos cambios no generan un impacto negativo (34).

Aspectos psicológicos. Existen diferencias en el impacto generado, pues se describe que las mujeres con prolapso han demostrado tener mayor prevalencia de depresión que mujeres sin esta condición, así mismo describen mayor impacto en la CV (43), sin embargo, otros estudios señalan que el prolapso genera poco efecto, en este sentido, aquellas que describieron alguna alteración refirieron falta de confianza, sentirse menos atractivas, menos femeninas o sentirse mal consigo mismas $(15,40)$. La incontinencia ha sido descrita como un tema privado para las mujeres (42).

Otros aspectos descritos, son problemas en la relación de pareja no orientada a actividad sexual (40) y en las relaciones sociales, por la preocupación que genera la pérdida de orina (42). Así, las pacientes con vejiga hiperactiva tienen más limitaciones sociales y emocionales que las mujeres sa- 
nas o con diabetes mellitus (41) y las mujeres con IO tienen conductas más evitativas (22).

También se describen alteraciones en el sueño relacionado a la incontinencia, ya sea por levantarse muchas veces o por incomodidad por tener un pañal o sentirse mojadas, además de tener sensación de cansancio hacia el final del día $(15,33)$.

Junto a los efectos psicosociales descritos, estudios señalan que la relación con los profesionales de salud podría ser un elemento que contribuya a los efectos negativos antes mencionados. Las mujeres refieren sentirse inseguras con la atención otorgada por el equipo de salud, describiendo ausencia de diálogo, falta de confianza, y falta de educación $(13,42)$. Así también se describe que los médicos no evalúan la incontinencia en las adultas mayores (33) y en este mismo sentido, las consultas a profesionales del área se realizan tardíamente $(33,34)$.

\section{DISCUSIÓN}

La presente revisión aporta a los profesionales del área una descripción de la vivencia de la mujer y de los aspectos mayormente afectados en su vida producto de las alteraciones de piso pélvico. Si bien la definición de la CV es compleja, la literatura concuerda en que incluye los aspectos físicos, psicológicos y sociales $(44,45)$, debido a que estas patologías tienen un gran impacto en los síntomas, el componente físico es el mayormente descrito.

A pesar de la descripción del impacto en la CV, existen controversias en cuanto a la severidad de este, lo que demuestra que la $\mathrm{CV}$ es un elemento difícil de evaluar. Barber (10), revisó los cuestionarios existentes en la literatura en relación a los trastornos de piso pélvico, recomendando seleccionarlos según el tipo de población a evaluar y las características psicométricas de cada uno. Creemos que lo vital es generar instrumentos validados adaptados a cada cultura que logren describir los efectos en la vida de la mujer para generar una intervención adecuada.

En relación a la etapa del ciclo vital, el impacto en la CV es similar en cuanto a síntomas, sin embargo, cada grupo experimenta diferentes efectos en la esfera psicosocial. La literatura coincide en que resulta importante apoyar a los adultos mayores a mejorar su CV con intervenciones específicas como educación en hábitos de eliminación (46-48). El impacto de la calidad de vida en pacientes adultos mayores con $\mathrm{IO}$, que viven en residencias de larga estadía, muestra resultados consistentes con las presentadas en esta revisión $(39,48)$. Otras intervenciones deben estar enfocadas durante la eta- pa de embarazo y puerperio generando estrategias de prevención y fortalecimiento del periné que han demostrado ser útiles para evitar estas alteraciones (49) facilitando por ejemplo el período expulsivo del proceso de parto.

Además de las intervenciones en el manejo de síntomas y en la prevención de las alteraciones, es necesario generar estrategias para disminuir los efectos psicosociales descritos. El equipo de salud tendría relevancia en generar confianza en las mujeres; esta evidencia se asemeja a lo descrito por mujeres en condiciones similares como lo es el proceso de ser histerectomizada en su dimensión social (50), por esto, es fundamental la necesidad de capacitar al equipo de salud en un enfoque integral, que incluya acogida, educación y resolución de dudas, apoyo emocional e incorpore la prevención en etapas precoces del ciclo vital.

Así mismo, el equipo de salud debe evaluar de manera específica las modificaciones en las actividades cotidianas, puesto que existen múltiples factores que podrían alterarlas y no necesariamente ligadas a las alteraciones de piso pélvico.

Respecto de la sexualidad, este es un tema que es posible de analizar de manera individual. Los resultados descritos coinciden con lo publicado en cuanto a función sexual para este grupo de mujeres como menor libido y menos orgasmos (51-53); en algunos estudios, se ha descrito que a pesar de las alteraciones, las mujeres tienen satisfacción en sus relaciones sexuales (52). Por esto, el enfoque de la mujer que presenta trastornos de piso pélvico, debe incorporar la evaluación de la función sexual, para poder entregar una atención integral centrada en todas las dimensiones de la calidad de vida.

Una de las limitaciones de esta revisión es que los artículos seleccionados analizan la CV de modo global, sin poder profundizar en algunas áreas como los efectos en la esfera sexual, o los efectos psicológicos de las mujeres. Otra limitación de la revisión es que describe los efectos de manera basal en las mujeres sin mediar intervenciones, lo que permite conocer cual es la realidad al realizar la primera consulta, pero no entrega información de los efectos posteriores a los tratamientos.

\section{CONCLUSIÓN}

Las mujeres con trastornos de piso pélvico describen un impacto negativo en varias dimensiones de la calidad de vida, destacando principalmente los aspectos físicos.

La evaluación de la calidad de vida, cada vez más es un resultado considerado en el éxito del tratamiento, lo que permite tener una aproximación 
objetiva del efecto de las intervenciones terapéuticas. Por esta razón, es necesario enfocar una atención integral a las mujeres, centrada en valoración de síntomas, efecto en la función sexual, educación y apoyo emocional.

\section{REFERENCIAS}

1. Haylen BT, de Ridder D, Freeman RM, Swift SE, Berghmans B, Lee J, et al. An International Urogynecological Association (IUGA)/International Continence Society (ICS) joint report on the terminology for female pelvic floor dysfunction. Neurourol Urodyn 2010;29:420.

2. Chen GD. Pelvic floor dysfunction in aging women. Taiwan J Obstet Gynecol 2007;46:374-8.

3. Swift S. Current opinion on the classification and definition of genital tract prolapse. Curr Opin Obstet Gynecol 2002;14:503-7.

4. Olsen AL, Smith VJ, Bergstrom JO, Colling JC, Clark AL. Epidemiology of surgically managed pelvic organ prolapse and urinary incontinence. Obstet Gynecol 1997;89:501-6.

5. Digesu GA, Santamato S, Khullar V, Santillo V, Digesu A, Cormio G, et al. Validation of an Italian version of the prolapse quality of life questionnaire. Eur J Obstet Gynecol Reprod Biol 2003;106:184-92.

6. Sung VW, Kauffman N, Raker CA, Myers DL, Clark MA. Validation of decision-making outcomes for female pelvic floor disorders. Am J Obstet Gynecol 2008;198:575.e1-575.e6.

7. Blaivas JG, Appell RA, Fantl JA, Leach G, McGuire EJ, Resnick NM, et al. Standards of efficacy for evaluation of treatment outcomes in urinary incontinence: recommendations of the Urodynamic Society. Neurourol Urodyn 1997; 16:145-7.

8. Barber MD, Walters MD, Bump RC. Short forms of two condition-specific quality-of-life questionnaires for women with pelvic floor disorders (PFDI-20 and PFIQ-7). Am J Obstet Gynecol 2005;193:103-13.

9. Wren PA, Janz NK, Brubaker L, Fitzgerald MP, Weber AM, LaPorte FB, et al. Reliability of health-related quality-of-life measures 1 year after surgical procedures for pelvic floor disorders. Am J Obstet Gynecol 2005;192:780-8.

10. Barber MD. Questionnaires for women with pelvic floor disorders. Int Urogynecol J Pelvic Floor Dysfunct 2007; 18:461-5.

11. Borello-France DF, Downey PA, Zyczynski HM, Rause CR. Continence and quality-of-life outcomes 6 months following an intensive pelvic-floor muscle exercise program for female stress urinary incontinence: a randomized trial comparing low- and high-frequency maintenance exercise. Phys Ther 2008;88:1545-53.

12. Caruso S, Bandiera S, Cavallaro A, Cianci S, Vitale SG, Rugolo S. Quality of life and sexual changes after double transobturator tension-free approach to treat severe cystocele. Eur J Obstet Gynecol Reprod Biol 2010 Jul;151:106-9.

13. Muller N. Pelvic organ prolapse: a patient-centred perspective on what women encounter seeking diag- nosis and treatment. AUST NZ CONTINENCE J 2010;16:70-80.

14. Borello-France DF, Handa VL, Brown MB, Goode $\mathrm{P}$, Kreder K, Scheufele LL, et al. Pelvic-floor muscle function in women with pelvic organ prolapse. Phys Ther 2007;87:399-407.

15. Srikrishna S, Robinson D, Cardozo L, Cartwright R. Experiences and expectations of women with urogenital prolapse: a quantitative and qualitative exploration. BJOG 2008;115:1362-8.

16. Fitzgerald MP, Janz NK, Wren PA, Wei JT, Weber $\mathrm{AM}$, Ghetti $\mathrm{C}$, et al. Prolapse severity, symptoms and impact on quality of life among women planning sacrocolpopexy. Int J Gynaecol Obstet 2007;98:24-8.

17. Fritel X, Varnoux N, Zins M, Breart G, Ringa V. Symptomatic pelvic organ prolapse at midlife, quality of life, and risk factors. Obstet Gynecol 2009;113:609-16.

18. Dooley $\mathrm{Y}$, Lowenstein L, Kenton K, FitzGerald M, Brubaker L. Mixed incontinence is more bothersome than pure incontinence subtypes. Int Urogynecol J Pelvic Floor Dysfunct 2008;19:1359-62.

19. Herrera Perez A, Arriagada Hernandez J, Gonzalez Espinoza C, Leppe Zamora J, Herrera Neira F. Quality of life and sexual function in postmenopausal women with urinary incontinence. Actas Urol Esp 2008;32:624-8.

20. Espuna Pons M, Puig Clota M. Lower urinary tract symptoms in women and impact on quality of life. Results of the application of the King's Health Questionnaire. Actas Urol Esp 2006 Jul;30:684-91.

21. Espuna Pons M, Puig Clota M. Coital urinary incontinence: impact on quality of life as measured by the King's Health Questionnaire. Int Urogynecol J Pelvic Floor Dysfunct 2008 May;19:621-5.

22. Kang Y, Phillips LR, Kim SS. Incontinence quality of life among Korean-American women. Urol Nurs 2010;30:130-6.

23. Tennstedt SL, Fitzgerald MP, Nager CW, Xu Y, Zimmern $P$, Kraus $S$, et al. Quality of life in women with stress urinary incontinence. Int Urogynecol J Pelvic Floor Dysfunct 2007;18:543-9.

24. Arano P, Rebollo P, Gonzalez-Segura Alsina D. Assessment of the health-related quality of life impact in female with mixed urinary incontinence. Actas Urol Esp 2009;33:410-5.

25. Frick AC, Huang AJ, Van den Eeden SK, Knight SK, Creasman JM, Yang J, et al. Mixed urinary incontinence: greater impact on quality of life. J Urol 2009;182:596-600.

26. Steinberg AC, Collins SA, O'Sullivan DM. The impact of flatal incontinence on quality of life. Am J Obstet Gynecol 2009;201:539.e1-539.e3.

27. Digesu GA, Chaliha C, Salvatore S, Hutchings A, KhuIlar $\mathrm{V}$. The relationship of vaginal prolapse severity to symptoms and quality of life. BJOG 2005;112:971-6.

28. Slieker-ten Hove MC, Pool-Goudzwaard AL, Eijkemans MJ, Steegers-Theunissen RP, Burger CW, Vierhout ME. Prevalence of double incontinence, risks and influence on quality of life in a general female population. Neurourol Urodyn 2010;29:545-50.

29. Kraus SR, Markland A, Chai TC, Stoddard A, FitzGerald MP, Leng W, et al. Race and ethnicity do not 
contribute to differences in preoperative urinary incontinence severity or symptom bother in women who undergo stress incontinence surgery. Am J Obstet Gynecol 2007;197:92.e1-92.e6.

30. Sung VW, West DS, Hernandez AL, Wheeler TL,2nd, Myers DL, Subak LL, et al. Association between urinary incontinence and depressive symptoms in overweight and obese women. Am J Obstet Gynecol 2009;200:557.e1-557.e5.

31. Richter HE, Kenton K, Huang L, Nygaard I, Kraus S, Whitcomb $\mathrm{E}$, et al. The impact of obesity on urinary incontinence symptoms, severity, urodynamic characteristics and quality of life. J Urol 2010;183:622-8.

32. Azuma R, Murakami K, Iwamoto M, Tanaka M, Saita $\mathrm{N}$, Abe $\mathrm{Y}$. Prevalence and risk factors of urinary incontinence and its influence on the quality of life of Japanese women. Nurs Health Sci 2008;10:151-8.

33. O'Dell KK, Jacelon C, Morse AN. 'I'd rather just go on as I am'--pelvic floor care preferences of frail, elderly women in residential care. Urol Nurs 2008;28:36-47.

34. Orlandi M, Azevedo S. Incontinência urinária e enveIhecimento: impacto no cotidiano e na qualidade de vida. Rev Bras Enferm 2009;62:51-6.

35. Kocaöz S, Talas MS, Atabekoğlu CS. Urinary incontinence in pregnant women and their quality of life S Kocaöz et al. Urinary incontinence, pregnancy and QOL. $\mathrm{J}$ Clin Nurs 2010;19:3314-23.

36. Pauls RN, Occhino JA, Dryfhout V, Karram MM. Effects of pregnancy on pelvic floor dysfunction and body image; a prospective study. Int Urogynecol J Pelvic Floor Dysfunct 2008;19:1495-501.

37. Espuna-Pons M, Puig-Clota M. Coital urinary incontinence. Associated symptoms and severity of incontinence. Actas Urol Esp 2009;33:801-5.

38. Zielinski R, Low LK, Tumbarello J, Miller JM. Body image and sexuality in women with pelvic organ prolapse. Urol Nurs 2009;29:239-46.

39. Oh HS, Kim MK, Seo WS. Effectiveness of a behavioral intervention program for urinary incontinence in a community setting. Taehan Kanho Hakhoe Chi 2005;35:1476-84.

40. Bradway C, Strumpf N. Seeking care: women's narratives concerning long-term urinary incontinence. Urol Nurs 2008;28:123-9.

41. Jelovsek JE, Barber MD. Women seeking treatment for advanced pelvic organ prolapse have decreased body image and quality of life. Am J Obstet Gynecol 2006;194:1455-61.

42. Zeznock DE, Gilje FL, Bradway C. Living with urinary incontinence: experiences of women from 'The last frontier'. Urol Nurs 2009;29:157-63.

43. Ghetti C, Lowder JL, Ellison R, Krohn MA, Moalli P. Depressive symptoms in women seeking surgery for pelvic organ prolapse. Int Urogynecol J 2010;21:855-60.

44. Haas BK. Clarification and integration of similar quality of life concepts. Image J Nurs Sch 1999;31:215-20.

45. Taylor RM, Gibson F, Franck LS. A concept analysis of health-related quality of life in young people with chronic illness. J Clin Nurs 2008;17:1823-33.

46. Aslan E, Beji NK, Erkan HA, Yalcin O, Gungor F. Urinary incontinence (UI) and quality of life (QoL) of the elderly residing in residential homes in Turkey. Arch Gerontol Geriatr 2009;49:304-10.

47. Malmstrom TK, Andresen EM, Wolinsky FD, Schootman M, Miller JP, Miller DK. Urinary and fecal incontinence and quality of life in African Americans. J Am Geriatr Soc 2010;58:1941-5.

48. Dubeau CE, Simon SE, Morris JN. The effect of urinary incontinence on quality of life in older nursing home residents. J Am Geriatr Soc 2006;54:1325-33.

49. Lee IS, Choi ES. Pelvic floor muscle exercise by biofeedback and electrical stimulation to reinforce the pelvic floor muscle after normal delivery. Taehan Kanho Hakhoe Chi 2006;36:1374-80.

50. Cerda P, Pino P, Urrutia M. Calidad de vida en mujeres histerectomizadas. Rev Chil Obstet Ginecol 2006;71:216-21.

51. Barber MD, Visco AG, Wyman JF, Fantl JA, Bump RC, Continence Program for Women Research Group. Sexual function in women with urinary incontinence and pelvic organ prolapse. Obstet Gynecol 2002;99:281-9.

52. Huang AJ, Subak LL, Thom DH, SK, Ragins AI, Kuppermann $M$, et al. Sexual function and aging in racially and ethnically diverse women. J Am Geriatr Soc 2009;57:1362-8.

53. Handa VL, Harvey L, Cundiff GW, Siddique SA, Kjerulff $\mathrm{KH}$. Sexual function among women with urinary incontinence and pelvic organ prolapse. Am J Obstet Gynecol 2004;191:751-6. 